\section{ORBITAL OPTIC NERVE SHEATH MENINGIOMA}

\section{SUMMARY \\ OPTIC NERVE ORBITAL MENINGIOMA}

Introduction: Orbital meningioma treatment has achieved significant success over the last period. Primary optic nerve sheath meningiomas by the clinically progressive finding, but still persistent sufficient visual acuity, can be treated by fractionated stereotactic radiotherapy. Surgery is indicated for secondary meningiomas of the orbit. Surgical treatment is indicated due to intracranial tumor propagation of meningioma from the chiasm towards the orbit.

Material and methods: In the period 2014 - 2016 we monitored in dispensary 15 patients with meningioma of the orbit, who were checked at least in yearly intervals and underwent magnetic resonance examination of the orbit and brain.

Results: In group of 15 patients with histologically unverified meningioma of the orbit, the surgical solution was indicated in 3 patients. In 14 (93.3\%) patients meningiomas were secondary infiltrating orbit from the intracranial part of visual pathways, and in 1 case meningioma was primary arising from the optic nerv.

We indicated enucleation and partial exenteration in $3(20 \%)$ patients. Histopathological examination confirmed meningioma - in two cases gr. I., in one patient gr. II. In all of them, more than 5 years after the primary diagnose of the process, the tumor infiltration from the chiasm towards the orbit was the indication for surgery. In one patient with meningioma gr. II in 12 months interval after surgery - exenteration with lid sparing technique, there was a further progression from the sella turcica area and the growth of tumor masses to the area of the orbital conus. Patient underwent secondary surgical reduction of tumor mass of the orbit and treatment with sandostatin.

Conclusion: When deciding to treat meningioma, it is necessary to involve multidisciplinary collaboration. Ophthalmology examination is important because further treatment is indicated on the basis of changes in visual function in correlation with the imaging methods. In cases of progression of the tumor with the infiltration of the orbit, resulting in the loss of visual acuity, in certain conditions a radical solution - enucleation with partial exenteration of the orbit, is necessary.

Key words: meningioma of the orbit, primary tumors of the orbit, secondary tumors of the orbit, enucleation, exenteration with lid sparing technique

Čes. a slov. Oftal., 74, 2018, No.1, p. 23-30

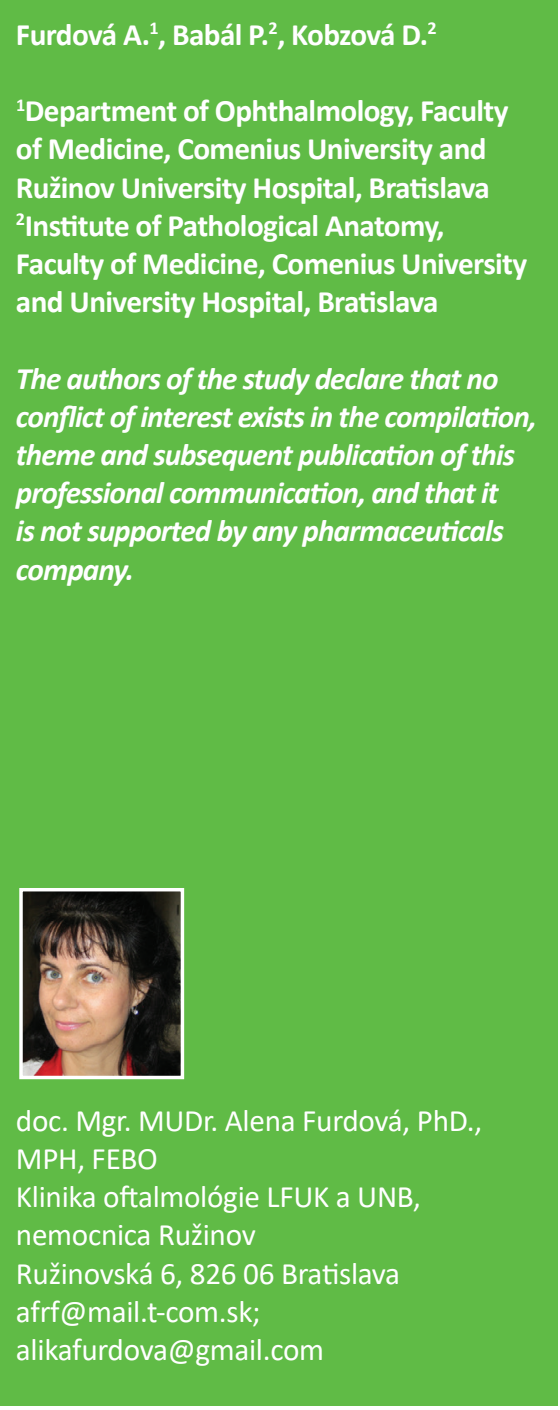

\section{INTRODUCTION}

Tumours of the orbit form a relatively rare and very heterogeneous group of tumorous expansions, primarily or secondarily infiltrating into the region of the orbit. In recent years, advances in radiodiagnostics, knowledge of the course of the pathology, radiosurgical techniques and modern chemotherapeutic protocols have changed the therapeutic procedures and the prognosis for these types of tumours [30].

The optimal procedure is therapy conducted by multidisciplinary teams in specialised centres, which deal with this issue. Meningiomas of the sheath of the optic nerve form only approximately $2 \%$ of all orbital tumours, and $1-2 \%$ of all meningiomas, but secondary meningiomas infiltrating into the orbit from the surrounding areas are found more frequently $[10,28]$.

Meningiomas appear more commonly in women in the age category of 30-50 years. However, meningioma may also be detected in children. That is to say that it can appear in every age category. The treatment of orbital meningioma has recorded marked successes in recent years. Primary meningiomas growing from the sheath of the optic nerve, in which the finding is clinically progressing but the patient's visual acuity still persists at an acceptable level, can be resolved by means of fractionated stereotactic radiotherapy. A surgical solution is indicated mainly in the case of secondary meningiomas of the orbit. Surgical treatment is indicated in the case of intracranial spread of a primary tumour or infiltration of a meningioma into the orbit from the region of the chiasm. When deciding on the procedure for treatment of meningioma, this always concerns multidisciplinary co-operation. An ophthalmological examination is important, because the further treatment is indicated on the basis of changes of visual functions, in correlation with imaging methods. In cases of progression of the tumour with infiltration of the orbit, where the result is a loss of visual functions, a radical solution is applied - enucleation to exenteration of the orbit. [11, 24, 31].

Primary orbital meningiomas of the sheath of the optic nerve originate from meningo endothelial cells of the arachnoid trabeculae of the dura mater of the optic nerve. As many as $92 \%$ of primary meningiomas of the sheath of the optic nerve begin from the intraorbital segment, but a small percentage start from the intracanalicular part [1,33]. Most frequently we encounter gr. I meningiomas according to the WHO classification [21].

Secondary meningiomas spread into the orbit from the in- 
tracanalicular region, originating primarily in the region of the planum sphenoidale or tuberculum sellae. Patients often seek medical attention in a stage when it is no longer possible to define precisely where the process primarily originated, whether in the region of the intraorbital part of the optic nerve and spreading intracanalicularly or vice versa. However, from a clinical perspective, this is not significant, a decision is taken on the further procedure after the patient has undergone an imaging examination and ophthalmological examination.

Bilateral meningiomas are very rare, occurring in less than $10 \%$ of cases [13]. They occur in younger middle age, more frequently in men [7]. Bilaterality is mostly linked with multiple tumours in the region of the brain [17], which often occur in patients with neurofibromatosis [6].

The majority of meningiomas in the orbital region cause slowly progressing neuropathy of the optic nerve, a deterioration of visual acuity, a constriction of the perimeter and disorders of colour vision. For meningiomas of the sheath of the optic nerve in an advanced stage, there is a pathognomonic triad (the Hoyt-Spencer triad) - progressive loss of vision, atrophy of the disc of the optic nerve and retinochoroidal (opticociliary) shunts. Ophthalmoscopically we may detect macular edema and choroidal folds. In the case of secondary tumours exophthalmos appears especially in the advanced stage of the pathology, as well as motility defects of the eyeball [26].

In differential diagnostics we must always consider optic nerve glioma, neuritis, granulomatous inflammation in the orbital region, lymphoma, leukaemic infiltrates and even melanoma [4].

We determine the diagnosis on the basis of imaging examinations in correlation with the clinical picture.

On computer tomography (CT) diffuse tubular, fusiform or globular enlargement of the optic nerve is displayed, with a hyperintensive sheath and central clarification [15]. The spread of ehtmoidal cells may also be a manifestation of intracanalicular meningioma [14].

On magnetic resonance imaging (MRI) meningioma of the sheath of the optic nerve is isointensive, with extraocular muscles in T1 balance and T2 balance. Separation of tumours is best observed in T1 balance with contrast (Gd-DTPA). This examination is essential for the determination of the diagnosis [18, 32].

We proceed with a biopsy by means of medial or lateral orbitotomy only in exceptional cases, because the radiological image of the meningioma of sheaths of the optic nerve is mostly sufficiently typical that such a biopsy is unnecessary.

Meningioma is a benign tumour, which does not metastasise and is not linked with increased mortality. It threatens in particular visual functions and upon a larger volume of the tumour mass in the orbit upon secondary infiltration, protrusion to lagophthalmos occurs, and may necessitate a more radical procedure.

\section{AIM}

To evaluate the results of the treatment of meningiomas in the period of 2014-2016 at the Department of Ophthalmology at the Faculty of Medicine of Comenius University and University Hospital in Bratislava.

\section{MATERIAL AND METHOD}

Cohort of patients at the Department of Ophthalmology at the Faculty of Medicine of Comenius University and University Hospital in Bratislava in the period of 2014 - 2016 with orbital meningioma. Indication for surgical solution in the cohort of patients.

\section{RESULTS}

In the period of 2014 - 2016 we observed and preventively monitored 15 patients with orbital meningioma at the Department of Ophthalmology of the Faculty of Medicine, Comenius University and University Hospital Bratislava. The average age of the patients was 58.3 years, with a predominance of women -11 (73.3\%). The patients were observed at annual intervals in outpatient care and underwent magnetic resonance imaging of the brain and orbit with a contrast substance.

In 14 (93\%) patients this concerned secondary meningiomas of the orbit infiltrating from the intracranial part of the visual pathway into the orbit. In only 1 patient this concerned a meningioma originating primarily from the sheath of the optic nerve in the orbit.

We indicated a surgical solution for $3(20 \%)$ patients, in whom an infiltration of a meningioma from the region of the chiasm into the orbit had taken place over the course of more than 5 years since the initial identification of the pathology, due to protrusion of the eyeball and worsening of the clinical finding. In all the patients the tumorous masses infiltrated across the optic canal from the region of the sella turcica. We indicated enucleation and partial exenteration with preservation of the eyelids for two women and one man.

In two patients a gr. I meningioma was confirmed by histopathological examination, in one patient a gr. II meningioma was confirmed.

Patient 1 -72-year-old woman, observed for more than 10 years, protrusion of the right eye occurred, indicated for enucleation of amaurotic painful eyeball.

Patient 2 - 65-year-old man, observed over the course of more than 15 years, in the last year with progression of the finding, with infiltration into the region of the sella turcica. We proceeded with enucleation of the amaurotic painful eyeball and partial exenteration of part of the tumorous masses in the orbit.

Patient 3 - 44-year-old woman following repeated radiotherapy in the past, in the last 3 years experienced worsening of finding in the orbital region of the left eye. With regard to the progression - protrusion of amaurotic eye with prolapse across the ocular aperture in 2016, we indicated enucleation and partial exenteration. During the course of the following months there was a further growth of tumorous masses within the region of the orbital conus. We indicated surgical reduction of the volume of the tumorous masses in the orbit. Meningioma gr. II was again histologically confirmed. The patient was referred for general therapy with Sandostatin, with a good local response. 
The details are presented in the case report.

\section{Case report}

The patient was observed from the year 2002 - condition following neurosurgical procedure for the following diagnosis: Tumour triloculare reg. intraorbitalis dx.et reg. suprasellaris et reg. chiasmatis. Histologically verified meningioma gr.1.

Progression of finding gradually from 2002. Radiotherapy repeatedly indicated: Condition after 1st series of IMRT (intensity-modulated radiation therapy) in a dose of 50 Gy / 2 Gy / 25x (treatment from 10.1. - 1.3.2007). Condition after 2 nd series of IMRT in a dose of $30 \mathrm{~Gy} / 2$ Gy / 15x . Treatment concluded 19.12.2013.

In 2015 the patient reported to our outpatient department due to progression of the finding in the right orbit. Protrusion on right side, for several months the patient had been unable to close the ocular aperture, progression of finding on cornea (Fig. 1, 2).

In January 2016 we indicated enucleation of the right eyeball and partial exenteration of the right orbit with preservation of the eyelids, due to progression of the intraorbital component of a complex extra-axial tumour (Fig. 3).

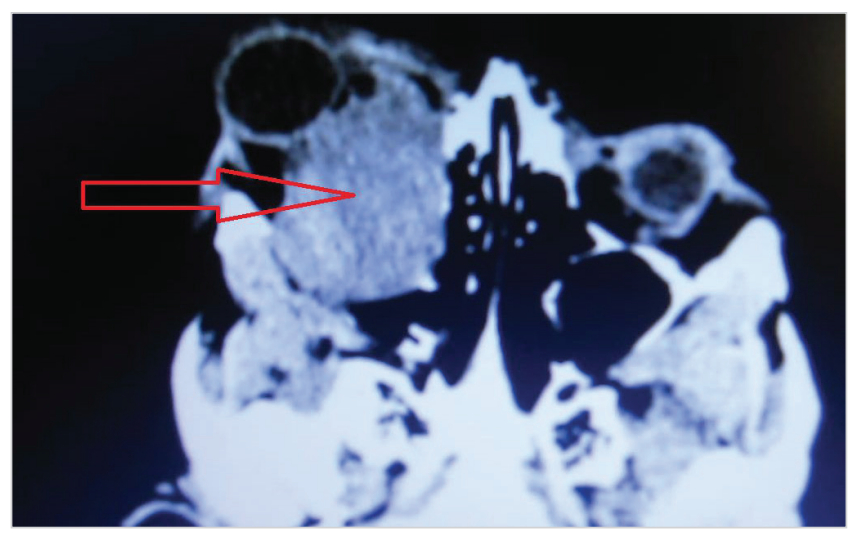

Fig. 1: MRI findings with tumor masses arising from chiasm to the orbit in $12 / 2015$
Histologically verified atypical meningioma gr. II according to WHO. Determined Ki67 30\% with increased mitotic activity. Healing took place without complications (Fig. 4).

Patient observed in outpatient care, gradual pronounced progression of the volume of tumorous masses in the orbit, without inflammatory infiltration, only mucous secretion in conjunctival sac (Fig. 5).

Gradual progression of tumorous masses in right orbit (Fig. 6).

The patient was sent for an MRI examination (4/2016) - confirmed pronounced progression of the intraorbital component of a three-lesion tumour, persistent complex extra-axial TU infiltrate localised predominantly in the right half of the neurocranium, spreading into the right orbit and infiltrating also into the foramen ovale - since the previous examination there had been pronounced progression of the part of the tumour localised in the orbit, now completely filling the entire orbit, which was furthermore expanded, in total the intraorbital part reached the size of approx. $58 \times 43 \times 46 \mathrm{~mm}$ (APxLLXCC); eyeball - condition after enucleation, raised part of TU stabilised since the last examination except for small nodule $T$ on right side (from $3.5 \mathrm{~mm}$ to $5.5 \mathrm{~mm}$ ).

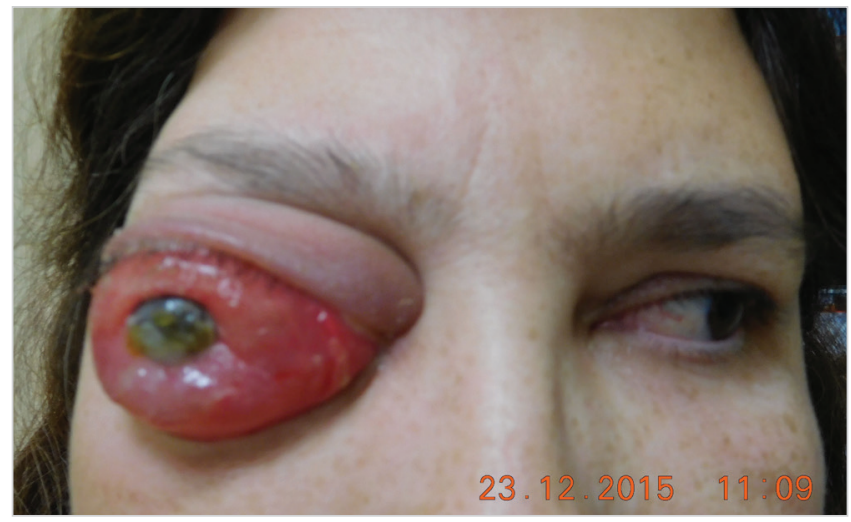

Fig. 2: Clinical finding in $12 / 2015$, eye globe prolabse through the eyelid, progression of corneal lesion
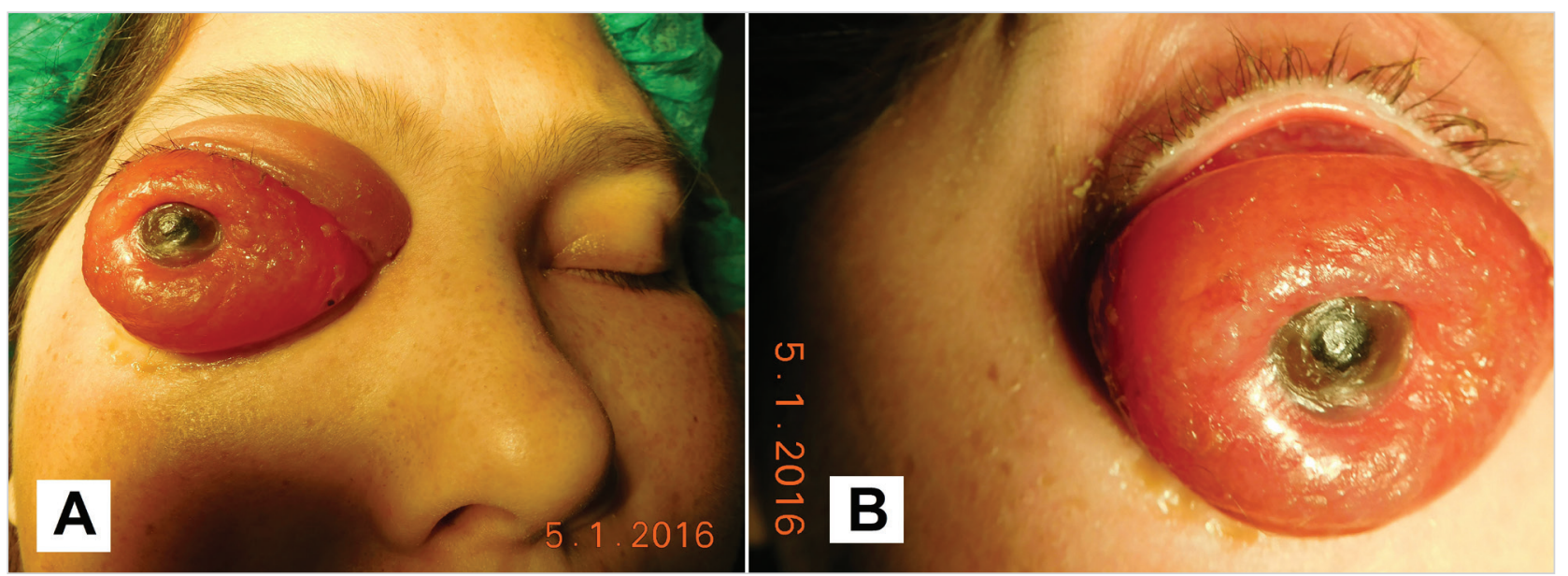

Fig. 3: Macrophoto $1 / 2016$ before surgery (A), detail of the eye globe (B) 

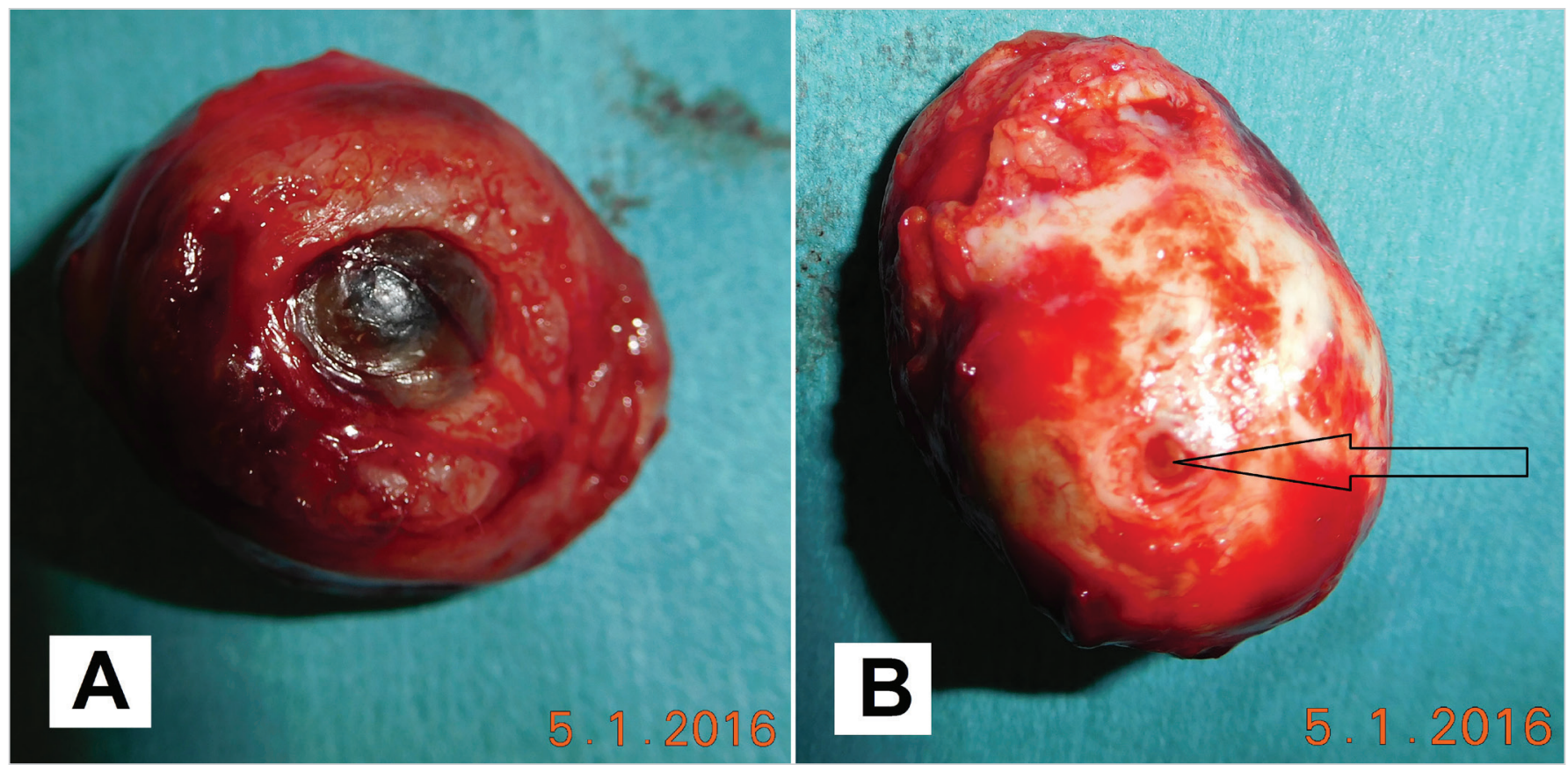

Fig. 4: $A, B$ Enucleated eye globe (A), the optic nerve is marked with the arrow (B)
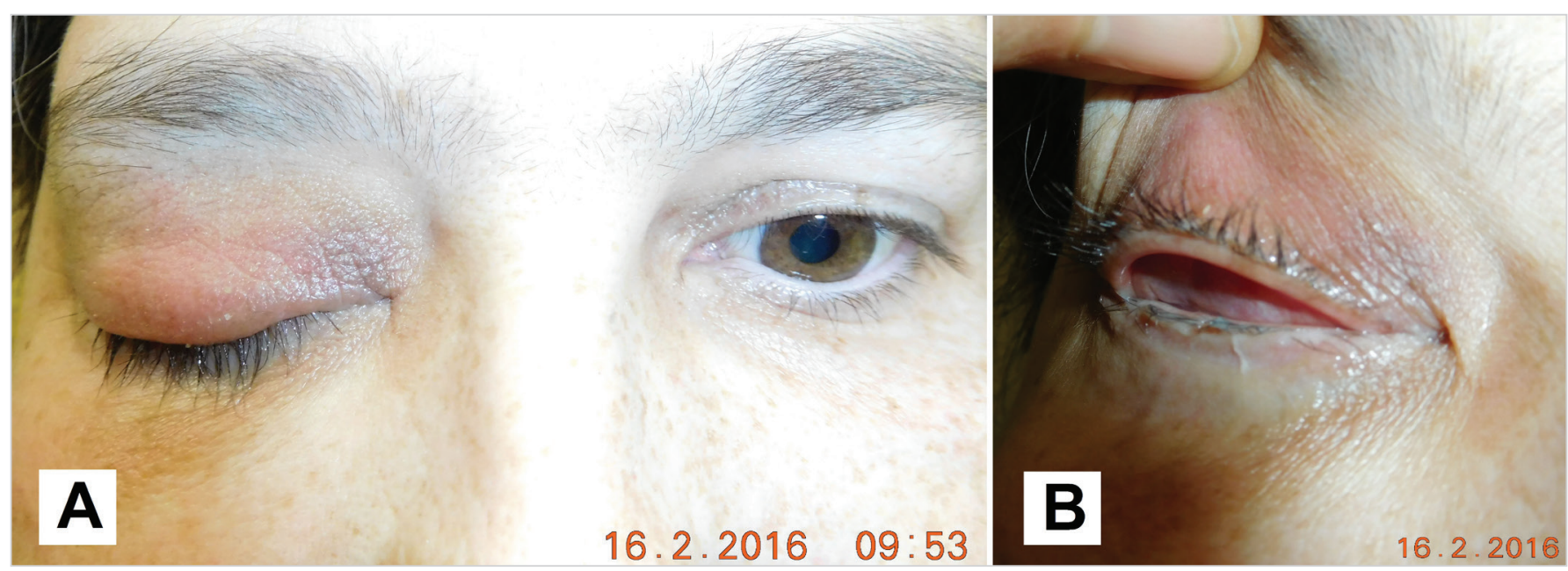

Fig. 5: Patient after the surgery $2 / 2016$, pseudoptosis (A), detail of the conjunctival sac (B)

We indicated a further full body examination with regard to the atypical meningioma reg. intraorbitalis a suprasellaris I. dx., following partial resection in 2002, following repeated RT (radiotherapy), following enucleation of right eyeball and exenteration of orbit 01/2016, with progressive growth postoperatively.

In 4/2016 full body Somatostatin receptor scintigraphy SRS - conclusion:

Radiopharmaceutical: 111In-octreotid i. v.; Applied activity: $185 \mathrm{MBq}$; Prístroj: Symbia T (Siemens).

Full body planar examination (anterior + posterior), tomographic SPECT/CT (native low dose protocol) of chest, abdomen and pelvis, $4 \mathrm{~h}$ and $24 \mathrm{~h}$ p.i.: gammagraphic highly intensive focal pathological uptake 111 In-octreotid in tissue filling right orbit, accumulation of fundamentally lower intensity also intracranially in middle cranial fossa. In other localities without detection of pathological focal uptake of radiopharmaceutical.

Physiological activity determined in kidneys, spleen, liver, excretion of RF by means of uropoetic system and enterally. Pathological tissue determined gammagraphically in right orbit, with very high intensity of expression of somatostatin receptors, intracrainally in middle cranial fossa pathological process with SR expression of medium intensity.

Subjectively the patient complained of pain behind the orbit and sometimes the right half fo the head. GeneraIly the patient has persistent spastic hemiparesis I. sin., without palpating signs of lymphatic infiltration in the region of the head. General therapy - analgesics, Diluran 1 tablet per day, famotidine $30 \mathrm{mg} 1$ tablet per day (Fig. 7).

The patient was presented to a commission, and according to the data from the literature, including NCCN in 2015 and phase II of clinical trials was given an order 


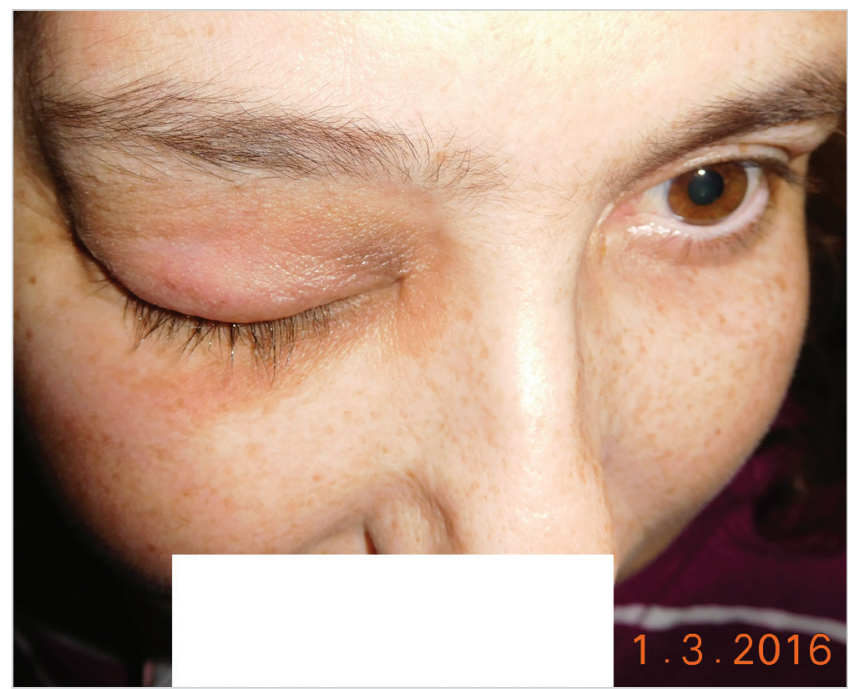

Fig. 6: Patient in 3/2016, eyelids are without inflammation

for an Octreoscan - urgent appointment. Subsequently in the case of sufficient positivity of the receptors, the proposed indicated therapy was SSA - SANDOSTATIN IAR $30 \mathrm{mg}$ for 28 days.

In the case of negativity the indicated therapy would be SUNITINIB 50 mg 28 days / 14 days off - all pharmaceuticals with the exception of the Ministry of Health.

The patient commenced treatment with sandostatin, with a subsequent markedly positive response in the region of the right orbit - local finding markedly improved,

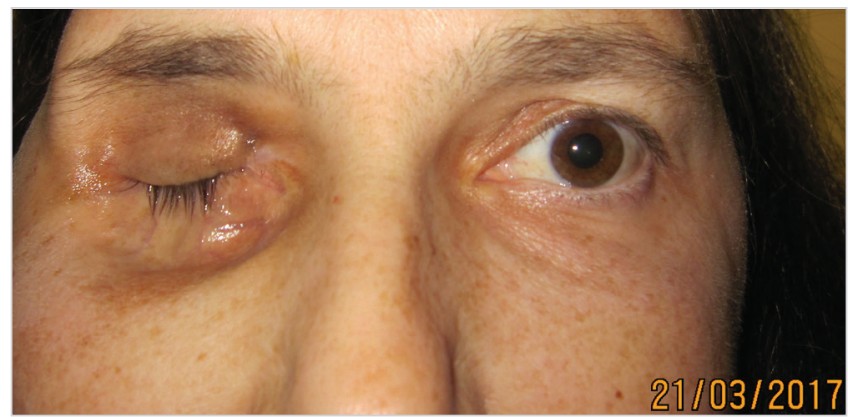

Fig. 9: Patient in 3/2017 after systemic therapy, regression of tumor findings in the orbit

eyelids pacific, without inflammatory reaction, without prolapse of tumorous matter, reduction of volume of tumorous masses in orbital conus (Fig. 8, 9).

\section{DISCUSSION}

Meningioma is a benign tumour, which does not metastasise and is not linked with increased mortality. Primary orbital meningioma threatens mainly visual functions, which usually worsen progressively over the course of the following years.

Despite all the difficulties in connection with the organisation of clinical trials on this theme, the treatment of meningioma of the optic nerve has undergone substantial changes. In the past patients were either observed or surgical excision of the tumour was performed, the latter
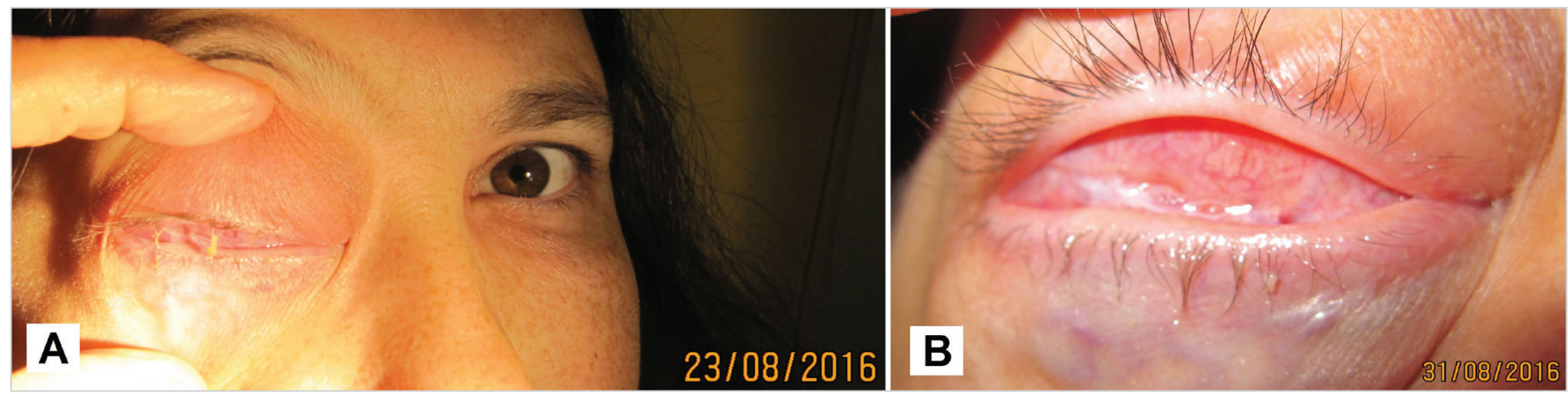

Fig. 7: Patient in 8/2016, progression of tumor masses in the right orbit (A), detail of conjunctival sac - conjunctiva with scar, symplepharon

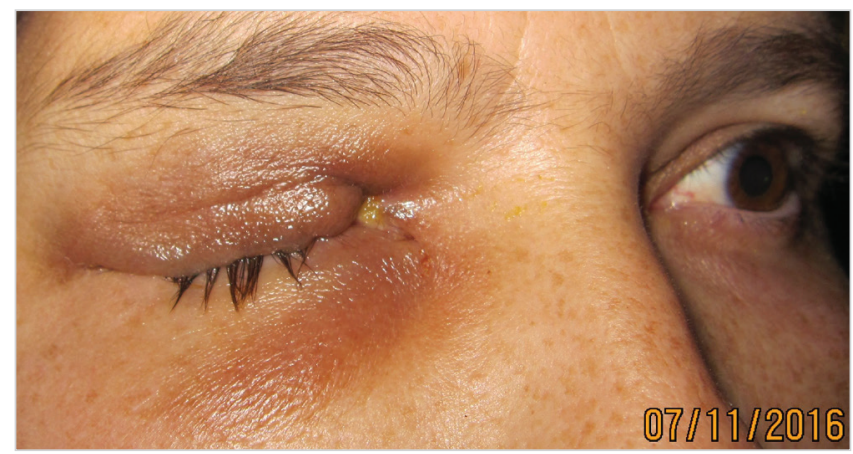

Fig. 8: Patient after reduction of tumor masses in the orbit in $11 / 2016$ of which nevertheless presented a high risk of blindness, malfunctions of the eyelids or motility of the eyeball. In the 1980 s the first successful attempts were made to perform radiotherapy on this type of tumour [16]. However, due to fears of radiation damage to the optics and surrounding tissues, the application of this therapeutic approach did not become standard until later. Ophthalmological examination of patients with primary meningioma of the optic nerve or secondary meningioma infiltrating into the orbit is of fundamental importance in deciding upon the following procedure. Changes of visual functions and their progression are decisive in the therapeutic procedure. 
The majority of retrospective studies have demonstrated that it is virtually impossible to remove meningioma completely. Subsequent ocular motility disorders and recurrences of the tumour appeared in 30\% of cases [22]. Surgical solution is recommended only in the case of intracranial spread of the tumour, poor central visual acuity of the affected eye and cosmetically obtrusive protrusion [24].

To date the results of treatment of meningiomas by chemotherapy are not well known. Meningioma cells often exprimate various hormone receptors, usually for progesterone or oestrogen. As a result they may be sensitive to treatment of their antagonists, but this has unfortunately not been confirmed. Different studies present different results of treatment. In a group of 16 recurrent meningiomas which did not influence the function of the optic nerve, treatment with somatostatin led in 10 cases to stabilisation or regression [8]. Mifepristone (Ru 486) was administered to 28 patients with meningioma not treatable by resection, and led to an improvement in eight of them [12], although in the second phase of the trial its effectiveness was not confirmed [9].

Radiotherapy is producing promising results in terms of preservation of sight and halting the growth of the tumour. Various techniques are available: 3D conformal radiation therapy, IMRT, stereotactic fractionated radiotherapy (SRT, SFR) and stereotactic radiosurgery (SRS) [20].

Kennerdell et al. [16] used fractionated radiotherapy on six patients. During the observation period of three to seven years, no complications occurred after exposure. In 2002 Turbín et al. [31] published a retrospective study with 64 patients with meningioma of the sheath of the optic nerve, who were either only observed or treated by surgical operation, operated on in combination with radiation or only irradiated. The observation period in this study was on average 150 months. The authors determined that the best long-term results were produced by treatment by radiation only, although in approximately one third of patients complications developed from radiation, such as radiation retinopathy, inflammation of the iris or atrophy of the temporal lobe. The study does not determine which type of radiotherapy was used on specific patients, but due to the long observation period the majority of patients were probably treated earlier by conventional rather than stereotactic techniques.

Saeed et al. [25] in their study on 34 patients treated with conventional or stereotactic fractionated radiotherapy with long-term observation (median 58 months) determined an improvement or stabilisation of central visual acuity in $91 \%$ of patients. Early complications up to six weeks after irradiation included hair loss and headaches. Within the framework of later complications, dry eye syndrome, cataract and radiation retinopathy were recorded. No difference was determined in the final effect on visual functions between conventional and stereotactic fractionated radiotherapy.

Arvold et al. [3] in their study on 25 patients (median of observation 30 months) treated by stereotactic photon or proton irradiation determined an improvement or stabilisa- tion of central visual acuity in $95 \%$ of patients, and a deterioration in only one of them, although this followed a prior improvement. Reitnopathy was recorded in three patients.

Baumert et al. [5] refer to a group of 23 patients in whom stabilisation or improvement of vision was improved in $95 \%$ of cases during the observation period (median 20 months). Improvement of vision was determined already within the course of one to three months following stereotactic fractionated radiotherapy. In one person there was a development of radiation retinopathy and vitreous haemorrhage four years after irradiation.

Conformal stereotactic fractionated radiotherapy (SRT, SFR) with immobilisation with the aid of fixation of a mask thus appears to be the optimal therapeutic method for the preservation or even improvement of vision in patients with a progressive or advanced primary meningioma of the sheath of the optic nerve and usable vision in the affected eye. It enables sufficient and targeted irradiation of the tumorous mass upon simultaneous minimal exposure of the surrounding tissues and minimisation of the adverse effects of irradiation. The total dose applied fractionated to the tumour is mostly within the range of 45 to $54 \mathrm{~Gy}$, the individual dose of irradiation of the optic nerve and chiasm upon a single application of radiosurgical irradiation shouId not exceed $8 \mathrm{~Gy}$. This technique requires complex planning, which is facilitated by special software and three-dimensional imaging of the region of the tumour [29].

Another possibility of radiotherapy is radiosurgical treatment by Leksell gamma knife, in the case of preservation of vision accelerated fractionation radiotherapy is possible, in which the stereotactic plan is divided into application over a period of five days. However, in the case of amaurosis, one-off stereotactic radiosurgical treatment is indicated.

Liu et al. [19] evaluated the effect of treatment by Leksell gamma knife in 30 patients with meningioma. The observation period was 56 months. Out of 30 patients vision improved in 11, central visual acuity remained unchanged in 13 (including patients with preoperative amaurosis), and deteriorated in six patients. Progression of the tumour was recorded in two cases.

Another possible alternative therapy is "frameless" robotic radiosurgery. A linear accelerator on the arm of a robot enables consistent application of simple or partial therapy [23].

In the case of a stale finding, we still incline toward the option of long-term observation of the patient and timely indication of irradiation if applicable. This applies for patients without significant clinical damage, without evidence of deterioration of vision or progression of an intracranial tumour. An ophthalmological examination should be conducted on these patients twice per year over the course of two to three years, and subsequently once per year once visual acuity is stable [20]. Follow-up CT or MR examinations are appropriate during the first and second year at 6 monthly intervals, over the course of the following two to three years always once per year and subsequently once per three to four years on the 
precondition that the clinical examination has not shown any progression $[2,5]$.

On the basis of the published clinical trials, stereotactic fractionated radiotherapy is used as the most effective treatment of first choice for progressive or advanced orbital meningioma on patients with good central visual acuity. This treatment is capable of halting the growth of the tumour and stabilising or even improving visual functions. Advances have also been enabled by constant technical improvements of both imaging methods and therapeutic procedures, in which the precision of irradiation of the target structures is being increased, thus minimising adverse effects on the surrounding tissues.

In the case of progression of a tumour and spread into the optic canal and higher sections, or upon infiltration of the tumour from the region of the sella turcica into the orbit, for an amaurotic, painful eye we indicate enucleation with partial exenteration of the orbit. In the case of our 3 patients we proceeded with a radical solution due to the protrusion of the eyeball, in which visual acuity on the affected side was on the level of questionable light perception. Exenteration with preservation of the eyelids is a suitable solution due to good postoperative healing. Treatment with sandostatin is indicated also for these types of tumours [27].

\section{CONCLUSION}

A problem of all studies focusing on the treatment of meningiomas, whether primary, originating from the sheath of the optic nerve, or secondary, which infiltrate into the orbit, is their rare incidence, their very slow progression, and therefore the need for long-term observation, the absence of histological verification of the finding in patients and the necessity of gathering data from a number of different centres. In the case of secondary meningiomas, which cause local complications in the orbital region, we proceed with a surgical solution and subsequent radiation therapy, if applicable in combination with chemotherapy.

\section{LITERATURE}

1. Alper, MG.: Management of primary optic nerve meningiomas. Current status--therapy in controversy. J. Clin. Neuroophthalmol. 1981;1(2):101-17.

2. Andrews, DW., Faroozan, R., Yang, BP., et al.: Fractionated stereotactic radiotherapy for the treatment of optic nerve sheath meningiomas: preliminary observations of 33 optic nerves in 30 patients with historical comparison to observation with or without prior surgery. Neurosurgery. 2002;51(4):890902; discussion 903-904.

3. Arvold, ND, Lessell, S., Bussiere, M., et al.: Visual outcome and tumor control after conformal radiotherapy for patients with optic nerve sheath meningioma. Int. J. Radiat. Oncol. Biol. Phys. 2009;75(4):1166-72.

4. Bains, S., Kim, U., Shanti, R.: Orbital melanoma with calcification: A diagnostic dilemma. Indian J. Ophthalmol. 2016;64(12):932-4.

5. Baumert, BG, Villà, S., Studer, G., et al.: Early improvements in vision after fractionated stereotactic radiotherapy for primary optic nerve sheath meningioma. Radiother. Oncol. 2004;72(2):169-74.

6. Bosch, MM, Boltshauser, E., Harpes, P., et al.: Ophthalmologic findings and long-term course in patients with neurofibromatosis type 2. Am. J. Ophthalmol. 2006;141(6):1068-77.

7. Bosch, MM., Wichmann, WW., Boltshauser, E., et al.: Optic nerve sheath meningiomas in patients with neurofibromatosis type 2. Arch. Ophthalmol. Chic. III 1960. 2006;124(3):379-85.
8. Chamberlain, MC., Glantz, MJ., Fadul, CE.: Recurrent meningioma: salvage therapy with long-acting somatostatin analogue. Neurology. 2007;69(10):969-73.

9. Chamberlain, MC, Tsao-Wei, DD, Groshen, S.: Salvage chemotherapy with CPT-11 for recurrent meningioma. J. Neurooncol. 2006;78(3):271-6.

10. Chynoranský, M., Furdová, A., Oláh, Z.: Exenteration of the orbit. Cesk. Oftalmol. 1994;50(2):92-7.

11. Dutton JJ.: Optic nerve gliomas and meningiomas.: Neurol. Clin. 1991;9(1):163-77.

12. Grunberg, SM, Weiss, MH, Russell, CA., et al.: Long-term administration of mifepristone (RU486): clinical tolerance during extended treatment of meningioma. Cancer Invest. 2006;24(8):72733.

13. Hart, WM., Burde, RM., Klingele, TG., et al.: Bilateral optic nerve sheath meningiomas. Arch. Ophthalmol. Chic. III 1960. 1980;98(1):149-51.

14. Hirst, LW., Miller, NR., Hodges, FJ., et al.: Sphenoid pneumosinus dilatans. A sign of meningioma originating in the optic canal. Neuroradiology. 1982;22(4):207-10.

15. Kanamalla US.: The optic nerve tram-track sign.: Radiology. 2003;227(3):718-9.

16. Kennerdell, JS., Maroon, JC., Malton, M., et al.: The Management of Optic Nerve Sheath Meningiomas. Am. J. Ophthalmol. 1988;106(4):450-7.

17. Lewis, T., Kingsley, D., Moseley, I.: Do bilateral optic nerve sheath meningiomas exist? Br. J. Neurosurg. 1991;5(1):13-8.
18. Lindblom, B., Truwit, CL., Hoyt, WF.: Optic nerve sheath meningioma. Definition of intraorbital, intracanalicular, and intracranial components with magnetic resonance imaging. Ophthalmology. 1992;99(4):560-6.

19. Liu, D., Xu, D., Zhang, Z., et al.: Longterm results of Gamma Knife surgery for optic nerve sheath meningioma. J. Neurosurg. 2010;113 Suppl:28-33.

20. Miller, NR.: New concepts in the diagnosis and management of optic nerve sheath meningioma. J. Neuro-Ophthalmol. Off. J. North Am. Neuro-Ophthalmol. Soc. 2006;26(3):200-8.

21. Nanda, A., Bir, SC., Maiti, TK., et al.: Relevance of Simpson grading system and recurrence-free survival after surgery for World Health Organization Grade I meningioma. J. Neurosurg. 2017;126(1):201-11.

22. Ringel, F., Cedzich, C., Schramm, J.: Microsurgical technique and results of a series of 63 spheno-orbital meningiomas. Neurosurgery. 2007;60(4 Suppl 2):214-221; discussion 221- 222.

23. Romanelli, P., Wowra, B., Muacevic, A.: Multisession CyberKnife radiosurgery for optic nerve sheath meningiomas. Neurosurg. Focus. 2007;23(6):E11.

24. Roser, F., Nakamura, M., Martini-Thomas, R., et al.: The role of surgery in meningiomas involving the optic nerve sheath. Clin. Neurol. Neurosurg. 2006;108(5):470-6.

25. Saeed, P., Blank, L., Selva, D., et al.: Primary radiotherapy in progressive optic nerve sheath meningiomas: a long- 
term follow-up study. Br. J. Ophthalmol. 2010;94(5):564-8.

26. Saeed, P., Rootman, J., Nugent, RA., et al.: Optic nerve sheath meningiomas. Ophthalmology. 2003;110(10):2019-30.

27. Schulz, C., Mathieu, R., Kunz, U., et al.: Treatment of unresectable skull base meningiomas with somatostatin analogs. Neurosurg. Focus. 2011;30(5):E11.

28. Shields, JA., Shields, CL., Scartozzi, R.: Survey of 1264 patients with orbital tumors and simulating lesions. Ophthalmology. 2004;111(5):997-1008.
29. Skorkovská, K., Kollová, A.: Meningeomy pochev zrakového nervu přehled současných léčebných možností. Čes. Slov. Neurol. Neurochir. 2012;75/108(4):420-5.

30. Tailor, TD., Gupta, D., Dalley, RW., et al.: Orbital neoplasms in adults: clinical, radiologic, and pathologic review. Radiogr. Rev. Publ. Radiol. Soc. N. Am. Inc. 2013;33(6):1739-58.

31. Turbin, RE., Thompson, CR., Kennerdell, JS., et al.: A long-term visual outcome comparison in patients with optic nerve sheath meningioma managed with observation, surgery, radiotherapy, or surgery and radiotherapy. Ophthalmology. 2002;109(5):890-899; discussion 899-900.

32. Vaněčková, M., Seidl, Z.: Možnosti zobrazení očního bulbu, orbity a optického nervu $v$ modalitě magnetické rezonance (MR). Neurol Praxi. 2006;7(3):164-7.

33. Wilson, WB.: Meningiomas of the anterior visual system. Surv. Ophthalmol. 1981;26(3):109-27. 